\title{
Implementing Dementia Care Mapping as a practice development tool in dementia care services: a systematic review
}

This article was published in the following Dove Press journal:

Clinical Interventions in Aging

\author{
Claire A Surr \\ Alys W Griffiths \\ Rachael Kelley \\ Centre for Dementia Research, \\ School of Health and Community \\ Studies, Leeds Beckett University, \\ Leeds, UK
}

Correspondence: Claire A Surr

Centre for Dementia Research, School of Health and Community Studies, Portland PD5I 2 City Campus, Leeds Beckett University, Leeds LSI 3HE, UK Tel +44 II38124316

Email c.a.surr@leedsbeckett.ac.uk

\begin{abstract}
Dementia Care Mapping (DCM) is an observational tool set within a practice development process. Following training in the method, DCM is implemented via a cyclic process of briefing staff, conducting mapping observations, data analysis and report preparation, feedback to staff and action planning. Recent controlled studies of DCM's efficacy have found heterogeneous results, and variability in DCM implementation has been indicated as a potential contributing factor. This review aimed to examine the primary research evidence on the processes and the barriers and facilitators to implementing DCM as a practice development method within formal dementia care settings. PUBMED, PsycINFO, CINAHL, The Cochrane Library-Cochrane reviews, HMIC (Ovid), Web of Science and Social Care Online were searched using the term "Dementia Care Mapping". Inclusion criterion was primary research studies in any formal dementia care settings where DCM was used as a practice development tool and which included discussion/critique of the implementation processes. Assessment of study quality was conducted using the Mixed Methods Appraisal Tool. Twelve papers were included in the review, representing nine research studies. The papers included discussion of various components of the DCM process, including mapper selection and preparation; mapping observations; data analysis, report writing and feedback; and action planning. However, robust evidence on requirements for successful implementation of these components was limited. Barriers and facilitators to mapping were also discussed. The review found some consensus that DCM is more likely to be successfully implemented if the right people are selected to be trained as mappers, with appropriate mapper preparation and ongoing support and with effective leadership for DCM within the implementing organization/unit and in organizations that already have a person-centered culture or ethos. Future development of the DCM tool should consider ways to save on time taken to conduct DCM cycles. More research to understand the ingredients for effective DCM implementation is needed.
\end{abstract}

Keywords: dementia, practice development, staff training, person-centered care

\section{Introduction}

Dementia Care Mapping (DCM) $)^{1,2}$ is an observational tool set within a practice development process that has been used for over 20 years to assist in the delivery of better quality formal care to people with dementia. It is a tool that has developed over time with feedback from practitioners, and the latest eighth edition was published in 2005, following a formal academic review, development and testing process. ${ }^{3}$ DCM is carried out by trained individuals known as mappers. To become a mapper requires attendance at a standardized 4-day course delivered by DCM trainers, licensed by the University of Bradford. The training course includes an assessment of competence in 
use of the tool. ${ }^{4}$ The DCM observational tool and practice development process are reported in detail elsewhere (see, eg, Brooker and $\mathrm{Surr}^{3}$ and $\mathrm{BSI}^{4}$ ); however, in brief, it involves a five-stage process including the following: the mappers briefing staff within the care setting about the process and what it involves; mapping, which is the process of observation and structured data collection; data analysis where the mappers produce summaries of the data ready to be shared with staff teams; feedback, where selected results of the mapping are fed back to provide staff and relatives with a picture of good practice and areas for development; and action planning, where the staff team works with the mappers to develop individual and unit level action plans for implementation. ${ }^{5}$

A number of systematic reviews have been published on DCM over the last 20 years. These include an early review of the methodological considerations and outcomes of studies using DCM as a practice development tool, ${ }^{6}$ a review of the research evidence on $\mathrm{DCM},{ }^{7}$ a review of its psychometric properties as a method for evaluating and enhancing ${ }^{8}$ care quality and a review of its efficacy in longterm care settings. ${ }^{9}$ Reviews of the psychometric properties of DCM have indicated some issues with reliability, validity and consistency in implementation of the tool. ${ }^{7,8}$ The studies on efficacy report heterogeneous results, with some positive results found for agitation, quality of life, falls and neuropsychiatric symptoms in care home residents with dementia and reduced stress and burnout in care home staff. ${ }^{7,9}$ Recent randomized controlled trials of DCM in care home settings have reported process and implementation issues as a potential explanatory factor for the disparities found in efficacy. ${ }^{10-12}$

The implementation of health-based interventions can be complex and there is often limited understanding of why these may or may not be effective. Potential issues can arise from the environment, people living with a health condition and health professionals. The importance of understanding the barriers and facilitators for implementation before undertaking such interventions has been emphasized. ${ }^{13}$ Generally, barriers and facilitators arise at different levels: organization, team and individual. ${ }^{14}$ The organizational context focuses on barriers and facilitators that arise in the practice environment, such as whether financial reimbursement is offered, lack of available time and the expectations of people living with health conditions. The team context focuses on current evidence and understanding, such as usual care practices and the priorities of leadership. The individual context focuses on the knowledge and attitudes of staff members, such as their sense of competence and understanding of the intervention and evidence. ${ }^{14}$
It is thought that interventions designed to target and change specific issues are more effective than those designed to make more general changes to practice. ${ }^{15}$ Within dementia care, many interventions have failed to show impact when compared to usual care. Those that have shown the greatest impact are tailored or personalized interventions, which take into account the needs of the person living with dementia and their family. ${ }^{16}$ Poor intervention delivery, including poor adherence to protocols, is a common barrier for implementation of psychosocial interventions, ${ }^{16}$ and the particular difficulties of implementing interventions in care homes are well established. ${ }^{17}$ Research evidence suggests that barriers to implementing dementia-focused interventions in care homes occur across organizational, team and individual contexts and include lack of staff confidence, lack of team cooperation and lack of time and resources to implement interventions, ${ }^{18}$ in line with research evidence about implementation issues across disciplines. ${ }^{14}$

To date, there have been few summaries of the evidence on the processes of implementation of DCM as a practice development tool. An edited book published in $2003^{19}$ reviewed various aspects of DCM implementation, based largely upon the experiences of the DCM expert contributors. A more recent 2010 British Standards Institute, Publicly Available Specification (PAS 800), ${ }^{4}$ provides a guide to implementing DCM within care provider organizations. However, to date, there have been no formal systematic reviews in this area. Given that understanding the features of effective DCM implementation and barriers and facilitators to this may support the design and implementation of future DCM research, a review of this nature is required. The aim of this review, therefore, was to examine the primary research evidence on the processes, barriers and facilitators to implementing DCM as a practice development method within formal dementia care settings.

\section{Materials and methods}

The review followed the seven mixed-methods systematic review steps as identified by Pluye and Hong. ${ }^{20}$

\section{Review questions}

The review aimed to answer the following questions:

- What is currently known about the implementation of DCM as a practice development intervention within formal dementia care settings?

- What are the barriers and facilitators to implementation of DCM in these settings? 


\section{Inclusion and exclusion criteria}

We included studies that met the following criterion: primary research study that used DCM as a practice development tool in any formal dementia care settings and included discussion/ critique of implementation processes.

We excluded studies if they

- Described the use of DCM, but did not critique implementation processes

- Used DCM as an outcome measure

- Reported only on the psychometric properties of DCM

- Were secondary research or personal views (eg, editorial, book review, systematic/literature review) and abstracts of communications or meetings

- Were a Master's thesis

- Used DCM in a non-dementia setting/with people who did not have dementia

- Reported on a methodological adaption of DCM not related to the processes of implementation as a practice development tool (eg, data collection using new technology, comparing maps of different lengths)

\section{Search strategies}

We searched PUBMED, PsycINFO, CINAHL, The Cochrane Library-Cochrane reviews, HMIC (Ovid), Web of Science and Social Care Online in August 2017 using the phrase "Dementia Care Mapping" for studies published in English with no restrictions on date of publication. Given Dementia Care Mapping is a standard, trademarked name for the tool, all studies using DCM as an intervention must use this phrase, and therefore, limiting the search terms to this ensured capture of all relevant studies, while minimizing identification of irrelevant studies. Reference lists of key papers and e-alerts were used to include papers published between search completion and October 2017.

\section{Study selection}

All references were downloaded into reference management software EndNote X7, ${ }^{21}$ and titles and abstracts of the studies were screened by the first author against the inclusion and exclusion criteria. Excluded studies were checked for agreement by the second author. The full papers of the remaining studies were then reviewed by the first and second authors and agreement reached on inclusion or exclusion against the criteria.

\section{Assessment of quality}

To ensure that all included studies had adequate methodological rigor, assessment of quality of the studies was conducted by the second and third authors independently using the Mixed Methods Appraisal Tool, ${ }^{22}$ which evaluates the methodological quality of research studies for the purpose of systematic reviews. Two screening criteria were used for all studies: 1) are there clear research questions or objectives? and 2) does the collected data address the research questions or objectives? If both of these screening criteria were marked as "yes", then quality assessment of the methodology was conducted. This consisted of four criteria for each methodology type (qualitative, quantitative randomized controlled, quantitative nonrandomized and quantitative descriptive), focusing on recruitment of participants, selection of appropriate methods, consideration of researcher influence and response rates. For mixed-methods studies, additional three criteria were included to identify whether a mixedmethods approach was appropriate, whether the qualitative and quantitative data were appropriately integrated and if consideration was given to the limitations of this approach.

Each methodological approach used within a paper was given a percentage score based on how many of the criteria were met (four criteria per method). In order to be included, studies were required to score a minimum quality rating of $75 \%$ for each method used.

\section{Data extraction and analysis}

Data were extracted from the papers using an extraction table. The standard stages of the DCM process were used as an a priori framework for data extraction, as these represent specific elements of the DCM process which need to be undertaken for a complete, successful DCM cycle. Additional extracted data that did not feature under one of the DCM stages were coded as either a barrier or a facilitator to DCM implementation and then analyzed using an inductive thematic analysis process to identify additional key themes related to successful or unsuccessful DCM implementation.

\section{Results}

A total of 822 papers were identified through searches and an additional four via other mechanisms before removal of duplicates (Figure 1). There were 332 duplicate items, leaving 494 records for screening. Of these, 423 were excluded at the title/abstract screening and a further 58 after full paper review, leaving 13 papers that were then reviewed using the quality assessment tool, resulting in one further paper being excluded.

\section{Study characteristics, design and quality}

The 12 included papers emanated from Australia $(n=3)$, the UK $(n=2)$, Germany $(n=3)$, the Netherlands $(n=1)$, 


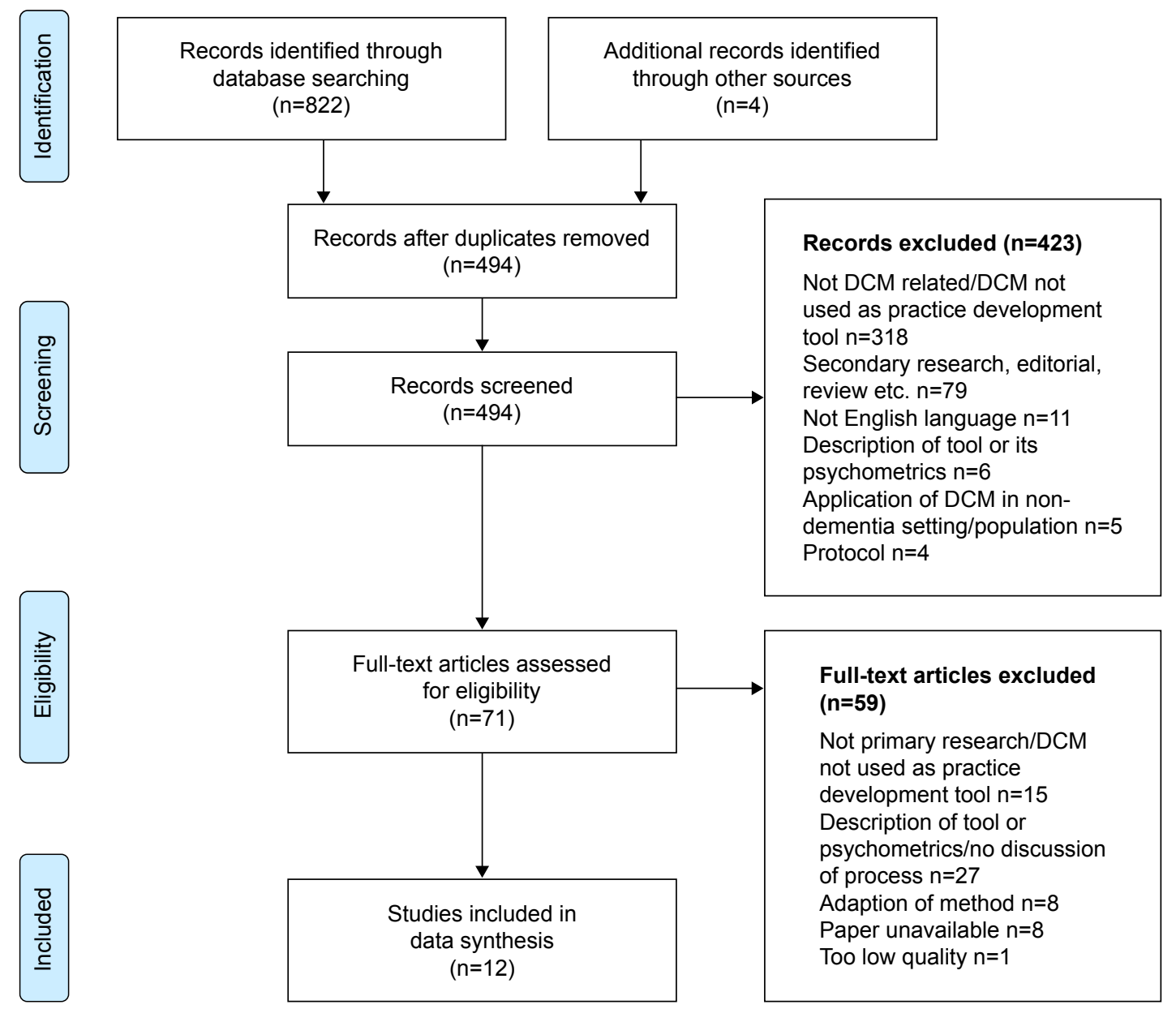

Figure I PRISMA flow diagram.

Abbreviations: DCM, Dementia Care Mapping; PRISMA, Preferred Reporting Items for Systematic Reviews and Meta-Analyses.

New Zealand $(n=1)$, Norway $(n=1)$ and the UK/USA $(n=1)$, representing a total of nine separate research studies (Table 1). Three of the studies represented in six papers ${ }^{12,23-27}$ were randomized controlled trials or adopted a quasi-experimental design with a control group, and aimed to examine the effectiveness of DCM as an intervention. Two studies were largescale surveys of DCM users ${ }^{28,29}$ and the remainder evaluated implementation of DCM in a single setting or organization. Seven of the studies implemented DCM in care home settings and two in a mental health hospital or National Health Service Mental Health Trust. Seven of the papers reported a formal evaluation of the DCM implementation process; in the other five studies, process issues were identified by the researchers in their discussion of DCM implementation in the project. Formal evaluation methods included surveys, ${ }^{28,29}$ reflective diaries, ${ }^{30}$ interviews and focus groups, ${ }^{25-27,31}$ questionnaires and documentary analysis. ${ }^{26,27}$ In five studies, there were no formal methods for evaluating DCM implementation or process issues; instead, these were detailed in the paper as part of the discussion and conclusion sections, based on the author(s) reflections on and critique of the implementation process. The quality check demonstrated that methodological rigor was generally high, although there were some issues with potential bias in recruitment, ${ }^{12,18,22}$ acceptable response rates ${ }^{20,21}$ and awareness of potential researcher influence. ${ }^{10,11,17,18,22}$

For the purposes of the review, we have structured the results around the different components of the DCM process.

\section{Mapper selection}

The majority of studies used a pragmatic design and trained staff from the setting as mappers who then implemented DCM within the research. However, in three studies, accounting for four papers, ${ }^{23,24,30,31}$ the mapping was led by the researchers or an external expert mapper with some or no input from trained mappers from the care home in which DCM was implemented. In one study where mappers from the care home were trained, ${ }^{12,25,26}$ a crossover approach was used with mappers from one care home delivering DCM in 


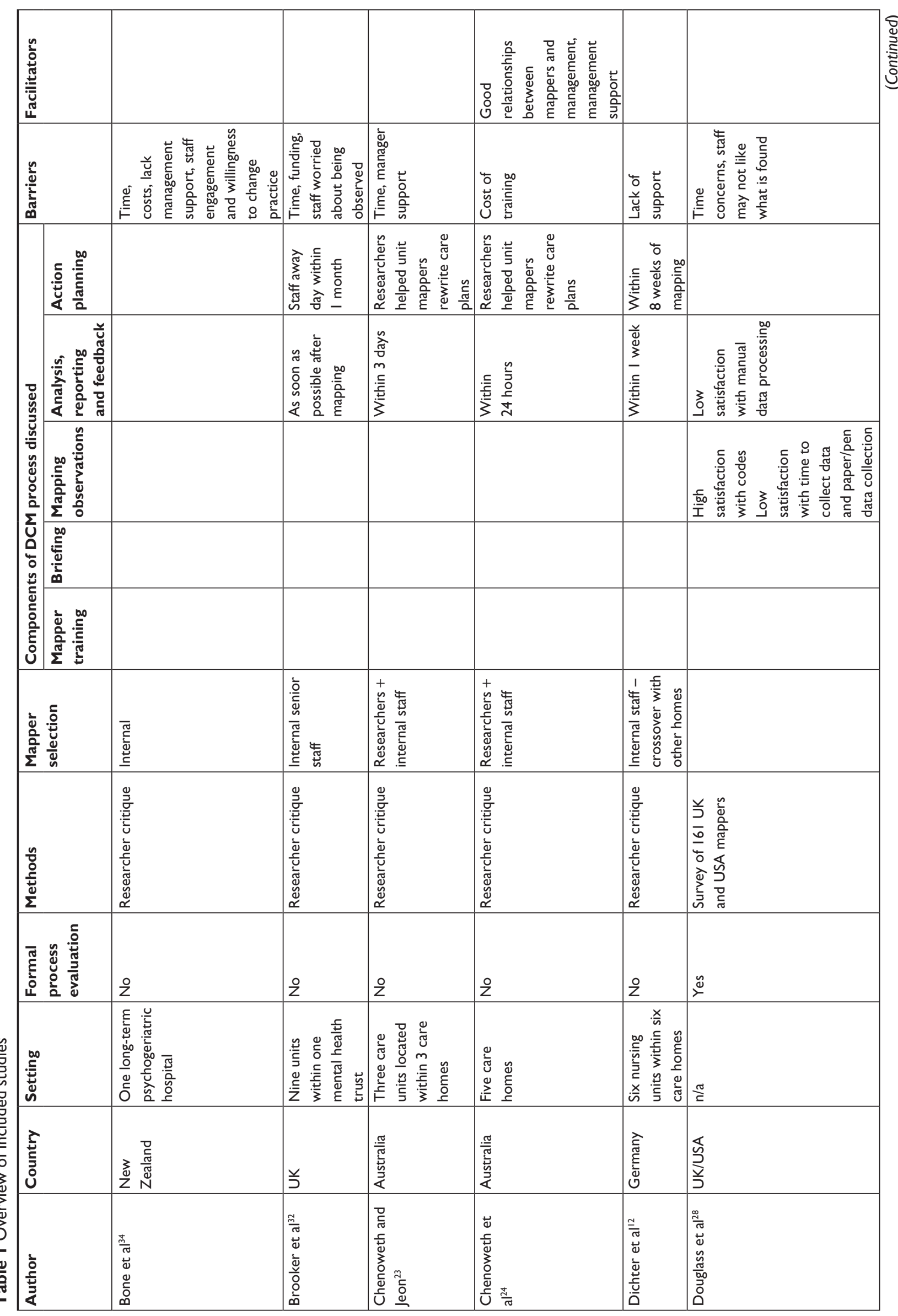




\begin{tabular}{|c|c|c|c|c|c|c|}
\hline \multirow{2}{*}{ 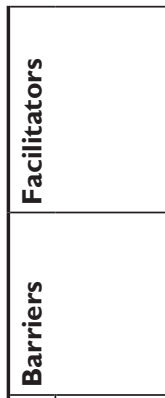 } & \multirow[b]{2}{*}{ 焉 } & \multirow[t]{2}{*}{ 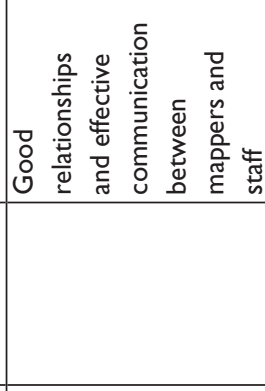 } & 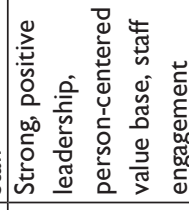 & \multirow{2}{*}{ 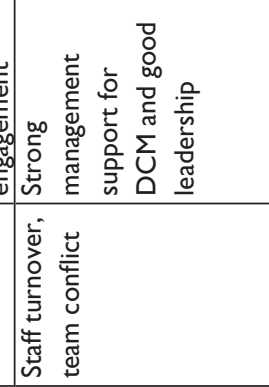 } & \multirow{2}{*}{ 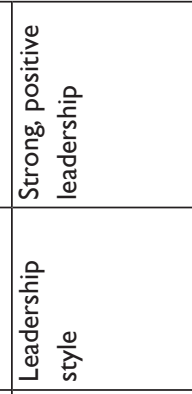 } & \multirow{2}{*}{ 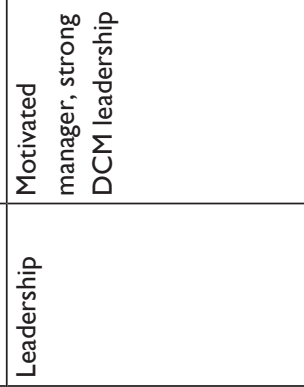 } \\
\hline & & & 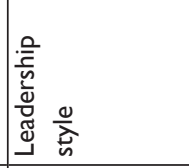 & & & \\
\hline 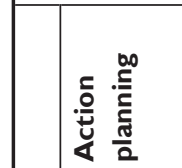 & & & & 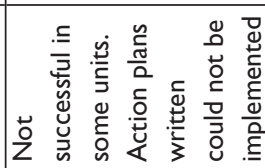 & & \\
\hline 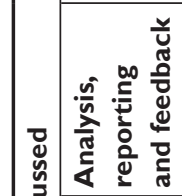 & & 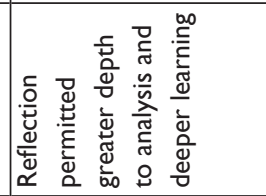 & & & & \\
\hline 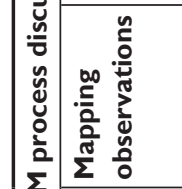 & & & & & & \\
\hline 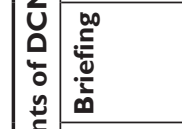 & & & & & & \\
\hline 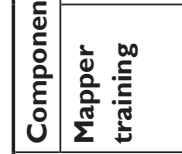 & & & & & & 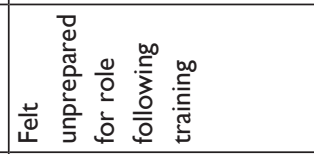 \\
\hline 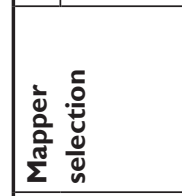 & 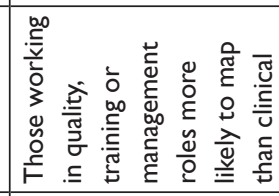 & 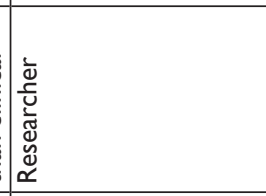 & 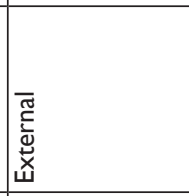 & 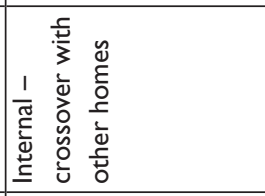 & 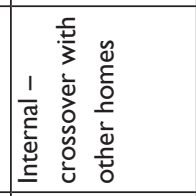 & 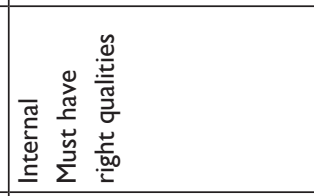 \\
\hline $\begin{array}{l}n \\
0 \\
0 \\
\vdots \\
0 \\
\Sigma \\
\end{array}$ & 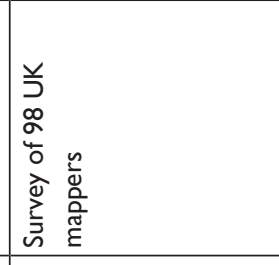 & 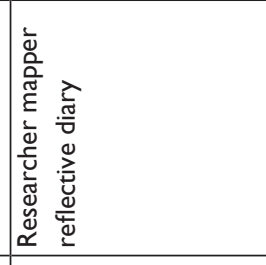 & 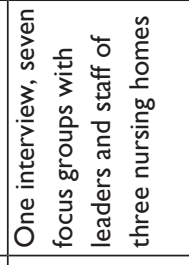 & 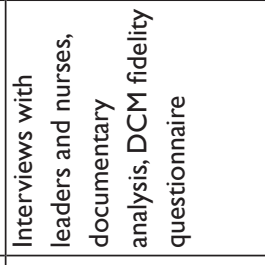 & 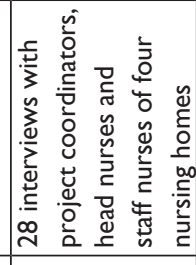 & 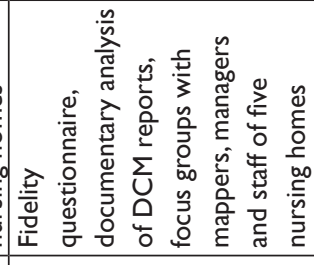 \\
\hline 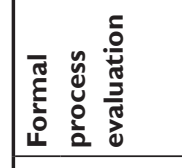 & $\stackrel{\mathscr{y}}{\check{\nu}}$ & $\stackrel{y}{x}$ & $\stackrel{y}{\rightleftharpoons}$ & $\stackrel{\circlearrowright}{\succ}$ & $y^{y}$ & $\stackrel{y}{\nu}$ \\
\hline 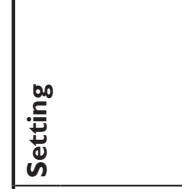 & $\frac{\pi}{\check{2}}$ & 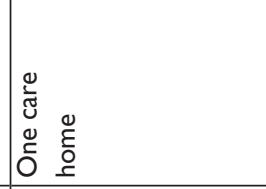 & 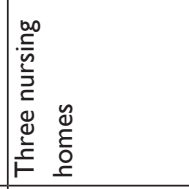 & 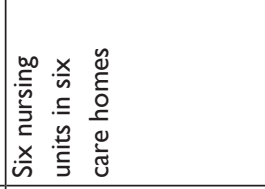 & 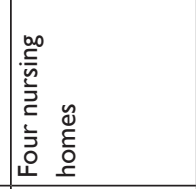 & 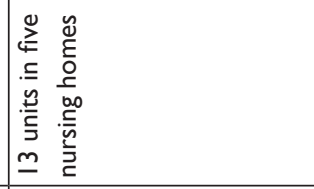 \\
\hline نे & 兰 & 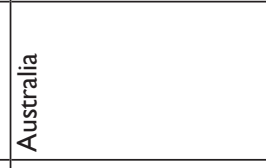 & 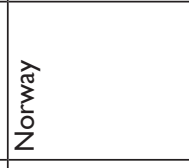 & 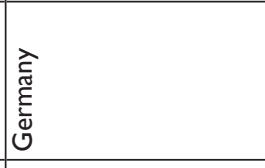 & 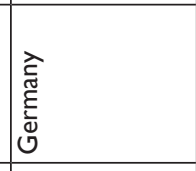 & 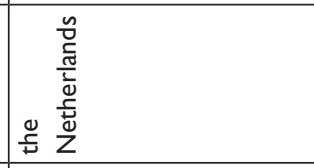 \\
\hline 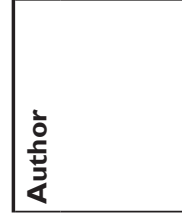 & 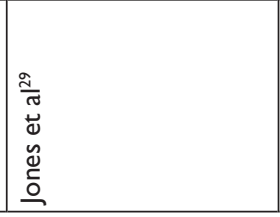 & 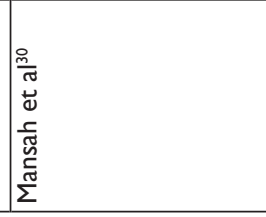 & 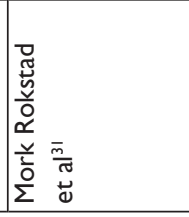 & 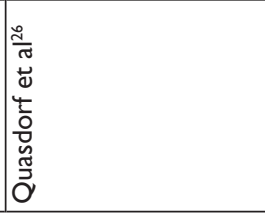 & 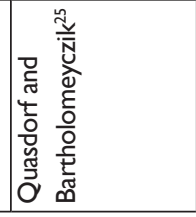 & 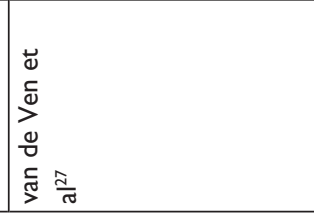 \\
\hline
\end{tabular}


one of the other participating sites. Two studies evaluated aspects of mapper selection. In the formal process evaluation conducted by van de Ven et al, ${ }^{27}$ focus group participants who had been involved in the implementation of DCM highlighted the importance of choosing a mapper with the right personality and skills. These skills included empathy, good communication skills, the ability to see the bigger picture and the ability to work successfully with teams including managing group processes and dealing effectively with resistance to feedback and change. Jones et $\mathrm{al}^{29}$ conducted a survey of trained mappers in the UK, asking respondents if they had implemented DCM post-training. They found that direct care/clinical staff were the least likely to have mapped, with less than half (45\%) of the respondents working in these roles having mapped since completing DCM training. Those working in quality and training roles were most likely to have mapped at $100 \%$ and $82 \%$ of respondents, respectively, with $62 \%$ of those in management roles and half of the researcher respondents stating they had completed at least one map since DCM training.

\section{DCM training and mapper preparedness}

Only one study discussed the DCM training course and how prepared mappers felt for their role post-training. Some mappers within the process evaluation conducted by van de Ven et $\mathrm{al}^{27}$ reported feeling unprepared for their role, despite completing the standard Basic User training as well as an additional 3-day Advanced DCM User course. Providing feedback to staff was described as a particularly anxietyprovoking element of the DCM process, and mappers identified a strong need for additional DCM training and support to use the tool effectively in practice. The authors highlight how, given that even advanced level training appears not to adequately prepare mappers to deliver DCM in the optimal manner, recruitment of mappers with the right competencies is crucial. They also recommend that the DCM training course is modified to include more content on delivering feedback, as well as being extended in length/depth to better prepare mappers for the role, taking into consideration their diverse skill and experience mix on entry to the course.

\section{Mapping observations}

Only one paper, which reported on surveys of mappers conducted in the UK and the USA, ${ }^{28}$ asked mappers about their experiences of conducting the observations and using the DCM coding frames. This found that mappers were generally satisfied with the coding frames used in DCM, although the US mappers were less likely to be satisfied with the coding of personal enhancers and detractors. However, the reasons for dissatisfaction were not recorded. Only $31 \%$ of the US mappers and $43.1 \%$ of the UK mappers were satisfied with the amount of time that it took to conduct observations and $43.1 \%$ of the US mappers and $66.7 \%$ of the UK mappers were satisfied with the paper and pen data collection methods. It should be noted that these surveys were conducted before the launch of the eighth edition of DCM in 2004, which included revisions to the coding of personal enhancers and detractors, ${ }^{3}$ and thus may have addressed some of the coding concerns.

\section{Data analysis, report writing and feedback} Only one study included any discussion of DCM data analysis and report writing. Douglass et $\mathrm{al}^{28}$ asked the US and UK mappers about their satisfaction with using a manual process for producing the DCM data analysis and reports and found low satisfaction with this $(27.6 \%$ and $31.9 \%$ satisfaction, respectively). Despite feedback being an area of concern raised by mappers when discussing preparedness to map, feedback processes and issues were rarely discussed in the papers included in the review. Four of the studies ${ }^{12,23,24,32}$ reported on the length of time they allowed between mapping and feedback in their respective mapping projects. As suggested by DCM guidance, ${ }^{33}$ which states feedback should be timely and within a month of observation, all study authors highlighted the need for feedback to occur quickly - time lapses between mapping and feedback in the studies ranged from 24 hours to 1 week. One process evaluation of a quasiexperimental study of DCM in care homes ${ }^{26}$ found that all but one of the six participating homes delivered a feedback session for each of the three cycles of mapping that took place. In two of the care homes, attendance of staff at feedback sessions was relatively low and in these units, staff ratings of the usefulness of feedback and the DCM process itself were negative. The staff were also critical of the quality of or the way in which DCM had been delivered by mappers, and therefore, staff reactions to DCM and engagement with the process appear to have a relationship with mapper skills and qualities. One study ${ }^{30}$ discussed the use of structured reflection within the mapping process, both for the mapper as part of the data analysis process and for staff during the feedback session and back in practice. The author concluded that the use of reflection permitted greater insight to occur during analysis and deeper learning through the opportunity to revisit reflections and create a shared community of reflective practice among staff.

\section{Action planning}

Action planning was also rarely discussed in any of the included papers, despite this being a crucial component for 
identification and implementation of practice change. Only two studies discussed how action planning was undertaken, with methods varying from a whole staff away day within a month of feedback ${ }^{32}$ to rewriting of care plans by researchers with the support of unit mappers. ${ }^{23,24}$ Another paper ${ }^{12}$ commented that action planning had to be completed within 8 weeks of observations, but gave no further details on the process for this. Two studies ${ }^{26,31}$ examining implementation of DCM in care home settings provided further analysis of the action planning process. Both studies identified that in around one-third of the care homes, action planning had been problematic or had not occurred. In two sites, no action plans were developed and, as a result, in one of these sites, the staff lost interest in participating in DCM. In another site, the action plans were unachievable and, therefore, could not be met. In units where action planning failed, staff ratings of the usefulness of the process were unsurprisingly negative.

\section{Facilitators of DCM implementation}

Six of the papers ${ }^{24-27,30,31}$ contained some analysis of factors the authors felt had been supportive of mapping. Key facilitators were effective communication between mappers and the staff on the unit being mapped, having a person responsible for organization and leadership of mapping, and management support for DCM. Quasdorf et $\mathrm{al}^{26}$ identified that strong champions for DCM at the team and management levels could ensure successful implementation of DCM even in the face of significant barriers. Two of the papers explored leadership of DCM in detail. Mork Rokstad et $\mathrm{al}^{31}$ identified that in nursing homes where DCM was implemented effectively, leaders used both transformational leadership, where a clear and coherent vision for person-centered care was presented, and situational leadership, which involved having a presence on the units and a sound knowledge of the staff teams and their skills. They also highlighted the need for leaders to be actively involved with resident care and to create a culture of shared responsibility for care quality with the staff team. Quasdorf and Bartholomeyczik ${ }^{25}$ contrasted the leadership styles across nursing homes that were either successful or unsuccessful at implementing DCM. Like Mork Rokstad et al, they found that in successful sites, there was strong leadership and the leaders presented a specific and clear vision for person-centered care. A clear organizational/unit vision for and ethos around person-centered care appeared particularly important for the success of DCM. In units where there was a dementia-friendly culture and staff had positive attitudes toward working with people with dementia, DCM was more likely to be successful. ${ }^{25,27}$

\section{Barriers to DCM}

Eleven of the 12 studies discussed barriers to DCM implementation. Barriers that were common across a number of studies included time (for training, mapping, feeding back and implementing changes), ${ }^{23,28,29,32,34}$ costs (of training and release of staff), ${ }^{24,32,34}$ lack of organizational and/or management support for DCM, ${ }^{23,25,27,29,31,34}$ staff resistance to change, ${ }^{27,28,34}$ organizational and management change (eg, change of manager, reorganization) ${ }^{25,27}$ and workload and staffing pressures. ${ }^{25,31}$ The barrier analyzed in greatest detail across the papers was leadership and management approaches. In contrast to the clear vision and the inclusive and engaging leadership styles of managers in care homes where DCM was successfully implemented, managers in homes where DCM was not a success were disengaged, unenthusiastic about DCM and were reluctant to lead or make decisions. ${ }^{25,31}$ Staff were often unclear whose responsibility DCM was. Managers who were perceived to be less supportive lacked a vision for good care or took a task-focused perspective, contrasting with the person-centered perspective of DCM. van de Ven et $\mathrm{al}^{27}$ found that manager choice around the use of DCM also had an impact on implementation. Managers who were enthusiastic about DCM and initiated participation in the research took responsibility to support the process, whereas managers who were told to take part in the research by their organization failed to buy into the process.

\section{Discussion}

This review has identified a dearth of primary research evidence on the implementation of DCM as a practice development tool within formal dementia care settings. Only 12 research papers have been published that undertake evaluation of DCM as a practice development tool and discuss the implementation issues, and only seven, representing six separate studies, included a formal evaluation of the experiences of those involved in DCM implementation. The majority of components of the DCM process were not considered within most of the 12 papers. Very few papers addressed mapper selection, training and preparedness for the mapping role, mapping observations, data analysis and report writing or feedback, and none examined briefing sessions. The majority of studies, however, identified some broad barriers or facilitators to DCM implementation. These correspond to individual, team and organizational contexts as identified in implementation science. ${ }^{14}$

At the individual level, mapper qualities and their ability to effectively lead DCM implementation were raised within 
a number of studies. There is a consensus among the studies discussing this area that selection of people who have the skills to implement and lead DCM is essential to successful implementation. Skills such as good communication, empathy and the ability to engage, work effectively with and provide leadership to staff teams were seen as crucial. Where mappers lacked these skills, this led to staff developing negative attitudes toward DCM and disengagement with the process.

Closely associated to mapper selection were the mapper perceptions of preparedness for their role following completion of DCM training. While this is a relatively unexplored area, the limited evidence from this review suggests that mappers felt that the DCM training they attended did not adequately prepare them to conduct all components of DCM; this was even the case, in one study, ${ }^{27}$ for mappers who had attended both Basic and Advanced User courses, equating to 6-7 days of training. While trained mappers generally appeared satisfied with use of the coding frames, ${ }^{28}$ the more complex and less structured components of the process such as feedback and action planning created greater anxiety and were less likely to be completed or completed well. ${ }^{26,27}$ Possible explanations for this are that the incorrect individuals were selected as mappers, and therefore, they did not already have the required underpinning communication and leadership skills. Alternatively, and more pragmatically, the health and social care workforce is heterogeneous, and therefore, any methods and their associated training provision must be able to account for varied previous experience, knowledge and skill levels. Therefore, DCM training may, at present, not be adequately preparing mappers to carry out the more complex components of the process, and as the authors of one study ${ }^{27}$ recommended, training may benefit from being longer and more in-depth to ensure individuals feel better prepared for what is a complex and challenging role. However, given research indicates that time is a significant concern for the majority of those attempting to implement DCM, adding additional time and costs to the training component seems unfeasible. In reality, any solution is likely to require a combination of appropriate selection of staff with the right skills and qualities, alongside consideration within the current DCM training program of how to ensure mappers feel better prepared to implement the method post-training.

At a team level, ability to be released from duties or make time available to implement DCM was identified as a major barrier to all components of mapping, with one of the survey studies ${ }^{29}$ indicating that mappers who had quality or training-focused roles were more likely to map than those in clinical or direct care roles. It may be that not only do the staff in these roles find it easier to find time within their usual work duties to undertake DCM, but they may also possess the skills as well as have the confidence to use the tool, because of the type of role they occupy. At a time of significant pressure on health and social care service delivery including staff shortages, it is perhaps unsurprising that staff in clinical or direct care roles find it more difficult to be released from day-to-day duties to undertake mapping.

Therefore, the evidence suggests that the role, personal qualities and skills of potential mappers must be considered when choosing who to train in DCM, as these influence the likelihood of mapping taking place and of DCM being implemented effectively. If less than half of the clinical staff trained actually go on to use DCM in practice, this raises questions regarding the cost-effectiveness of training them in the method.

At an organizational level, time for application was a general concern across all areas of DCM implementation, including training, mapping and then effecting practice change. Addressing this issue is likely to be challenging, since studies identified how poor implementation of the DCM process, including failure to adequately engage staff teams, poor or no feedback and lack of or poorly developed action plans, resulted in staff dissatisfaction and a breakdown of the process. Therefore, to be completed well, the evidence suggests DCM requires adequate time to be given to each part of the process and failure to do so is likely to lead to a failure to support effective practice change. There are a range of potentially time-saving components that could be introduced to reduce, for example, data analysis and report preparation processes, which were a particular area of dissatisfaction for some mappers. ${ }^{28}$ These include electronic data collection and storage via, for example, a computer or tablet device, and automated data processing and production of a feedback report. Some developments have been made in this area, including introduction of an Excel-based program to support automated generation of the scores and graphs for DCM reporting and, more recently, an online database system with automatic report generation facilities. ${ }^{35}$ While both currently require manual data inputting, the latter of these has the potential to support electronic DCM data collection in the future. Despite the known high turnover rates of staff in health and social care settings internationally, ${ }^{36-40}$ staff turnover was mentioned as a barrier to DCM implementation in only one study. ${ }^{26}$ In this study, DCM was more successfully implemented in units with teams that were more stable. One unit, however, managed successful implementation despite 
high staff turnover and several changes in the head nurse during the project period. This was said by the authors to be mitigated by stability from a project coordinator who led the implementation process.

The area about which is most written currently with regard to DCM implementation is leadership. It is clear from the available evidence that successful implementation or not of an individual DCM cycle and of sustained use of the method over time, all hinges on how effective the leadership and managerial support for DCM is. Studies included in this review identified key leadership qualities required for effective DCM implementation; these included strong leadership both for DCM and within the unit in which it is implemented. Leaders that were successful in supporting DCM implementation were "transformational", presenting a clear and coherent vision, culture and an ethos that valued and aspired to the delivery of person-centered care. They were also "situated" or present within the care home, engaging with staff and having a strong presence on the unit. These findings mirror those of other studies examining effective leadership approaches and styles in the context of culture change in care home and health care settings. ${ }^{41-43}$

A final, interesting issue raised by the research evidence on DCM implementation is the need for DCM to be implemented in organizations that already have a culture and ethos that embraces person-centered care. This suggests that rather than being a method that can take a setting from a task-focused and non-person-centered culture to one of person-centered practice, in fact, DCM appears to be a tool that is better placed to support services that have already made the decision to shift their culture, to find evidence-based ways to achieve this. In this sense DCM appears not to be a useful tool for converting services to embrace a personcentered culture, but a method for supporting those who already aspire to delivery of person-centered care to achieve their full potential. This indicates then that DCM may not be suitable for everyone, and may be only likely to work for services if adopted at the right time.

Comparing DCM to the 73 implementation strategies identified by Waltz et al, ${ }^{44}$ as a tool and process it encompasses many of the strategies across the nine identified strategy clusters, including: use of evaluative and iterative strategies such as having an implementation blueprint and utilizing cyclical small tests of change; provision, in some cases, of interactive assistance through supervision and support by a more experienced user; and training and education of stakeholders, including specialist training for mappers and briefing of staff implicit within the process. Areas where
DCM, however, is weaker with regard to key implementation strategies include areas such as clear assessment of readiness for DCM and identification of barriers and facilitators; provision of centralized technical assistance; the ability to adapt and tailor to the context; preparation of and engagement with organizations ahead of DCM training and implementation; engagement of stakeholders such as people with dementia and family members as active participants in the process; and the utilization of financial strategies to support implementation. A particular feature missing from implementation strategies for DCM then is to work with organizations to assess readiness, identify barriers and facilitators, and to support them to set up a team and wider organizational environment where DCM is likely to succeed. DCM training is provided at the individual level with usually two or more staff from an organization trained. They are then expected to go on to not only implement the tool and process to effect practice change, but also to lead team and organizational change to support use of the DCM tool in the first instance. These issues are in line with research evidence that barriers to implementing dementia-focused interventions in care homes often occur at an organizational or team level, and that support in these areas is key to successful implementation.

Bringing together all of the above evidence, this review indicates that careful consideration of the organizational context and readiness for DCM is, therefore, required ahead of commencing its use. The requirement of organizational readiness for change has been identified as critical in the literature ${ }^{45}$ Considering Scaccia et al's ${ }^{45}$ model for organizational readiness of, motivation to implement, and general organizational capacities and innovation-specific capacities to adopt the intervention, while the published DCM implementation guideline ${ }^{4}$ discusses organizational context, the DCM tool and implementation process itself currently lacks consideration of this. Therefore, the evidence from published research to date, combined with implementation science theory suggests that any service hoping to achieve benefit from DCM will need to aspire to delivering person-centered dementia care, and this needs to be a commitment throughout all staff including senior and local managers, ancillary staff and those in direct care roles. The service needs someone in place already who will provide strong and positive leadership for DCM and trained mappers who have the skills, qualities and time to implement it correctly. In some of the studies, DCM was implemented by external mappers who were either researchers or expert practitioners not employed by the organization. Using this model may offer some benefits in terms of costs as well as reducing the challenges associated with 
release of staff from usual duties to carry out DCM. It may also support the use of mappers with appropriate skills and knowledge, given the diversity of the health and social care workforce and the lack of confidence mappers report in using DCM after training. Future research might investigate the feasibility of different models of mapper selection and mapping and their impact on implementation, as well as the setting conditions or process required to create those, ahead of DCM implementation, which are likely to support its successful use.

The evidence from the published studies suggests that appropriate time needs to be dedicated to all stages of the DCM cycle, including a commitment to write and implement realistic action plans. DCM is unlikely to be a method than can solve the problems of a failing service, which has weak leadership and no vision or real commitment to implement it. While controlled studies of DCM's efficacy have to date found heterogeneous results in terms of outcomes for people with dementia, care staff and services, a number of these studies have raised implementation issues as a potential explanation for the lack of efficacy observed. Studies where DCM was implemented by researchers rather than internal mappers showed significant positive changes on outcomes such as quality of life and resident agitation, ${ }^{12}$ and lack of adequate implementation of some components of DCM was found where care staff led the process. ${ }^{26,27}$ This may be related to mapper skills and preparedness as well as team and organizational issues such as time and the ability to be released from normal work duties to implement DCM. The limited evidence available for inclusion in this review suggests that DCM is not an easy or simple option, or a quick fix solution to problematic care. Instead, we recommend that services wishing to implement DCM need to seriously consider their preparedness for it and willingness to engage with the process of change, in order to increase their chances of successful and sustained implementation.

\section{Limitations}

This review has a number of limitations. We only included studies published in English and, therefore, may have excluded additional evidence published in non-English journals. We only included primary research studies in our review and excluded a number of publications that were based on practitioner descriptions of their experiences of DCM implementation, with no formal evaluation. While we wished to ensure only evidence of at least a minimum quality level was included in this review, there are likely to be some useful, albeit anecdotal insights, in this larger body of practice evidence.
Our review was based on a limited number of papers, reflecting a smaller number of studies of which fewer still had conducted a formal process evaluation of DCM implementation. Our findings and conclusions are, therefore, based on a limited evidence base and more research is needed to better understand the components required for effective DCM implementation and the barriers and facilitators to implementation. One randomized controlled trial including an integrated process evaluation is currently underway; ${ }^{46}$ however, further robust research is needed on DCM implementation.

\section{Conclusion}

Despite DCM being used in practice for over 20 years and the growing body of research evidence around its use, there is relatively little evidence on how the approach is applied in practice. The available evidence suggests there are certain organizational features and contexts that are likely to be required for successful and sustained use of DCM, in particular, good leadership, organizational and management support and mappers who have the qualities, skills and time to undertake such a role. However, further research is required for a fuller understanding of what needs to be in place for effective implementation of DCM. Given that DCM is a resource-intensive tool with varying reports of efficacy from clinical trials, potentially due to variable implementation, further research into the processes and contexts required to ensure successful implementation is required to ensure that investment in DCM is not wasted. Future DCM implementation studies should include detailed process evaluations in order to better understand implementation issues and provide strategies to help overcome these.

\section{Disclosure}

CAS has conducted research on DCM and its use, including the development of the current eighth edition of the tool, and has previously been an approved DCM trainer. The authors report no other conflicts of interest in this work.

\section{References}

1. Bradford Dementia Group. Evaluating Dementia Care. The DCM Method. 7th ed. Bradford: University of Bradford; 1997.

2. Bradford Dementia Group. DCM 8 User's Manual. Bradford: University of Bradford; 2005.

3. Brooker D, Surr C. Dementia Care Mapping (DCM): initial validation of DCM 8 in UK field trials. Int J Ger Psychiatry. 2006;21(11): 1018-1025.

4. BSI. PAS 800:2010. Use of Dementia Care Mapping for improved person-centred care in a care provider organization. Guide. London BSI; 2010.

5. Brooker D, Surr C. Dementia Care Mapping: Principles and Practice. Bradford: University of Bradford; 2005. 
6. Beavis D, Simpson S, Graham I. A literature review of dementia care mapping: methodological considerations and efficacy. J Psych Ment Health Nurs. 2002;9(6):725-736.

7. Brooker D. Dementia care mapping: a review of the research literature. Gerontologist. 2005;45(1):11-18.

8. Cooke HA, Chaudhury H. An examination of the psychometric properties and efficacy of Dementia Care Mapping. Dementia (London). 2013;12(6):790-805.

9. Barbosa A, Lord K, Blighe A, Mountain G. Dementia Care Mapping in long-term care settings: a systematic review of the evidence. Int Psychogeriatr. 2017;29(10):1609-1618.

10. Mork Rokstad AM, Røsvik J, Kirkevold Ø, Selbaek G, Benth JS, Engedal K. The effect of person-centred dementia care to prevent agitation and other neuropsychiatric symptoms and enhance quality of life in nursing home patients: a 10-month randomized controlled trial. Dement Geriatr Cogn Disord. 2013;36(5-6):340-353.

11. van de Ven G, Draskovic I, Adang EM, et al. Effects of dementia-care mapping on residents and staff of care homes: a pragmatic clusterrandomised controlled trial. PLoS One. 2013;8(7):e67325.

12. Dichter MN, Quasdorf T, Schwab CGG, et al. Dementia care mapping: effects on residents' quality of life and challenging behavior in German nursing homes. A quasi-experimental trial. Int Psychogeriatr. 2015; 27(11):1875-1892.

13. Grol R. Personal paper. Beliefs and evidence in changing clinical practice. BMJ. 1997;315(7105):418-421.

14. Oxman A, Flottorp S. An overview of strategies to promote implementation of evidence-based health care. In: Silagy C, Haines A, editors. Evidence-Based Practice in Primary Care. Vol 2nd ed. London: BMJ Books; 2001.

15. Grol R, Grimshaw J. From best evidence to best practice: effective implementation of change in patients' care. Lancet. 2003;362(9391): 1225-1230.

16. Olazaran J, Reisberg B, Clare L, et al. Nonpharmacological therapies in Alzheimer's disease: a systematic review of efficacy. Dem Geriatr Cogn Disord. 2010;30(2):161-178.

17. Iliffe S, Manthorpe J, Warner J, et al. Making progress in psychosocial research in dementia. Dementia. 2008;7(2):167-174.

18. Rapaport P, Livingston G, Murray J, Mulla A, Cooper C. Systematic review of the effective components of psychosocial interventions delivered by care home staff to people with dementia. BMJOpen. 2017; $7(2): \mathrm{e} 014177$.

19. Innes A. Dementia Care Mapping: Applications Across Cultures. Baltimore, MD, US: Health Professions Press; 2003.

20. Pluye P, Hong QN. Combining the power of stories and the power of numbers: mixed methods research and mixed studies reviews. Ann Review Public Health. 2014;35:29-45.

21. EndNote $X 7$ [computer program]. Thomas Reuters; 2015.

22. Souto R, Khanassov V, Hong QN, Bush P, Vedel I, Pluye P. Systematic mixed studies reviews: updating results on the reliability and efficiency of the mixed methods appraisal tool. Int J Nurs Stud. 2015;52(1): $500-501$.

23. Chenoweth L, Jeon YH. Determining the efficacy of Dementia care mapping as an outcome measure and a process for change: a pilot study. Aging Ment Health. 2007;11(3):237-245.

24. Chenoweth L, King MT, Jeon YH, et al. Caring for aged dementia care resident study (CADRES) of person-centred care, dementia-care mapping, and usual care in dementia: a cluster-randomised trial. Lancet Neurol. 2009;8(4):317-325.

25. Quasdorf T, Bartholomeyczik S. Influence of leadership on implementing Dementia Care Mapping: a multiple case study. Dementia (London). 2017:1471301217734477

26. Quasdorf T, Riesner C, Dichter MN, Dortmann O, Bartholomeyczik S, Halek M. Implementing dementia care mapping to develop personcentred care: results of a process evaluation within the Leben-QD II trial. J Clin Nurs. 2017;26(5-6):751-765.
27. van de Ven G, Draskovic I, Brouwer F, et al. Dementia care mapping in nursing homes: a process evaluation. In: Van de Ven G, editor. Effectiveness and Costs of Dementia Care Mapping Intervention in Dutch Nursing Homes. Nijmegen, Netherlands: Radboud University Nijmegen; 2014.

28. Douglass C, Keddie A, Brooker D, Surr C. Cross-Cultural Comparison of the Perceptions and Experiences of Dementia Care Mapping "Mappers" in the United States and the United Kingdom. J Aging Health. 2010;22(5):567-588.

29. Jones S, Hamilton J, Surr C. Monitoring and improving the quality of person-centred care in health and social settings using Dementia Care Mapping (DCM): staff experiences of implementation barriers and supports. Eur J Pers Cent Healthc. 2016;4(1):1-7.

30. Mansah M, Coulon L, Brown P. A mapper's reflection on Dementia Care Mapping with older residents living in a nursing home. Int J Older People Nurs. 2008;3(2):113-120.

31. Mork Rokstad AM, Vatne S, Engedal K, Selbæk G. The role of leadership in the implementation of person-centred care using Dementia Care Mapping: a study in three nursing homes. J Nurs Manag. 2015;23(1): 15-26.

32. Brooker D, Foster N, Banner A, Payne M, Jackson L. The efficacy of Dementia Care Mapping as an audit tool: report of a 3-year British NHS evaluation. Aging Ment Health. 1998;2(1):60-70.

33. Bradford Dementia Group. Dementia Care Mapping: Process and Application. Bradford: University of Bradford; 2014.

34. Bone C, Cheung G, Wade B. Evaluating person centred care and Dementia Care Mapping in a psychogeriatric hospital in New Zealand: a pilot study. $N Z$ J Occup Ther. 2010;57(1):35-40.

35. Surr C, Hamilton J, Firth $P$, et al. Monitoring and improvement using Dementia Care Mapping. J Dement Care. 2015;23(5).

36. Skills for Care. The state of the adult social care sector and workforce in England. Leeds: Skills for Care; 2017.

37. Kovner CT, Brewer CS, Fatehi F, Jun J. What does nurse turnover rate mean and what is the rate? Policy Polit Nurs Pract. 2014;15(3-4): 64-71.

38. Imison C, Bohmer R. NHS and Social Care Workforce: Meeting Our Needs Now and in the Future? London: The King's Fund; 2013.

39. WHO. The World Health Report 2006-Working Together for Health. Geneva: WHO; 2006

40. Duffield CM, Roche MA, Homer C, Buchan J, Dimitrelis S. A comparative review of nurse turnover rates and costs across countries. J Adv Nurs. 2014;70(12):2703-2712.

41. Corazzini K, Twersky J, White HK, et al. Implementing culture change in nursing homes: an adaptive leadership framework. Gerontologist. 2014;55(4):616-627.

42. West MA, Lyubovnikova J, Eckert R, Denis JL. Collective leadership for cultures of high quality health care. J Organ Effectiveness People Perform. 2014;1(3):240-260.

43. Dixon-Woods M, Baker R, Charles K, et al. Culture and behaviour in the English National Health Service: overview of lessons from a large multimethod study. BMJ Qual Saf. 2013;23(2):106-115.

44. Waltz TJ, Powell BJ, Matthieu MM, et al. Use of concept mapping to characterize relationships among implementation strategies and assess their feasibility and importance: results from the Expert Recommendations for Implementing Change (ERIC) study. Implement Sci. 2015;10:109.

45. Scaccia JP, Cook BS, Lamont A, et al. A practical implementation science heuristic for organizational readiness: $\mathrm{R}=\mathrm{MC}^{2}$. J Community Psychol. 2015;43(4):484-501.

46. Surr CA, Walwyn R, Lilley-Kelly A, et al. Evaluating the effectiveness and cost-effectiveness of Dementia Care Mapping ${ }^{\mathrm{TM}}$ to enable personcentred care for people with dementia and their carers (DCM-EPIC) in care homes: study protocol for a randomised controlled trial. Trials. 2016;17(1):300. 
Clinical Interventions in Aging

\section{Publish your work in this journal}

Clinical Interventions in Aging is an international, peer-reviewed journal focusing on evidence-based reports on the value or lack thereof of treatments intended to prevent or delay the onset of maladaptive correlates of aging in human beings. This journal is indexed on PubMed Central, MedLine,

CAS, Scopus and the Elsevier Bibliographic databases. The manuscript management system is completely online and includes a very quick and fair peer-review system, which is all easy to use. Visit http://www.dovepress. $\mathrm{com} /$ testimonials.php to read real quotes from published authors.

Submit your manuscript here: http://www.dovepress.com/clinical-interventions-in-aging-journal 